\title{
Leucemia linfoblástica aguda en niños con síndrome de Down: análisis comparativo con pacientes sin síndrome de Down
} Acute lymphoblastic leukemia in children with Down syndrome:
Comparative analysis versus patients without Down syndrome

Méd. Carla L. Pennella ${ }^{a}$ Dr. Jorge G. Rossib , Bioq. Edgardo M. Baialardoc, Dra. Cristina N. Alonso , Méd. Myriam R. Guitter ${ }^{a}$, Méd. Cristian G. Sánchez La Rosaa, Méd. Natalia C. Millán ${ }^{a}$, Méd. Elizabeth M. Alfaro ${ }^{a}$ Dr. Pedro A. Zubizarreta y Dra. María S. Felice. ${ }^{a}$

\section{RESUMEN}

Introducción: Los niños con síndrome de Down (SD) tienen mayor frecuencia de leucemia linfoblástica aguda (LLA) y menor supervivencia que pacientes sin síndrome de Down (NSD). Analizamos las características clínicas, demográficas-biológicas y respuestas al tratamiento en SD-LLA versus NSD-LLA. Pacientes y métodos: Pacientes (0-19 años) con LLA desde enero de 1990 a noviembre de 2016. Se compararon características demográficas biológicas y respuestas al tratamiento con chi cuadrado y Wilcoxon rank sum. La supervivencia global y el intervalo libre de eventos (ILE) se analizaron con Kaplan-Meier y el test log-rank. Resultados: Se incluyeron 1795 pacientes, 54 con SD. Los SD-LLA presentaron edad mayor $(\mathrm{p}=$ 0,0189 ). Todosinmuno fenotipo precursor- $\mathrm{B}$, con menor incidencia de anomalías recurrentes $(\mathrm{p}<$ $0,0001)$. Demostraron mejor tasa de respuesta a prednisona $(p=0,09)$ y mayor mortalidad en inducción y remisión completa $(\mathrm{p}<0,0001)$. Todas las muertes de los SD-LLA fueron relacionadas con el tratamiento. La sobrevida libre de eventos en niños SD-LLA vs.NSD-LLA fue $47( \pm 8) \%$ vs. $73( \pm 1) \%(\mathrm{p}=0,006)$ y el ILE de los SD-LLA vs. NSD-LLA fue $54( \pm 9) \%$ vs. $75( \pm 1) \%(p=0,0297)$. La tasa de recaídas fue similar en ambos grupos $(\mathrm{p}=0,6894)$. El ILE de los SD-LLA fue menor en el grupo de 6-9 años: $39( \pm 19) \%(\mathrm{p}=0,7885)$. Conclusiones: Los niños de 6-9 años con SD-LLA años presentó menor sobrevida. Aunque estos niños presentaron una mejor respuesta temprana, la sobrevida libre de eventos e ILE fueron menores debido a la mortalidad relacionada con el tratamiento.

Palabras clave: leucemialinfoblásticaaguda, niños, sindrome de Down, pronóstico.

http:/ / dx.doi.org/10.5546/aap.2018.e500

Texto completo en inglés:

http:/ / dx.doi.org/10.5546/ aap.2018.eng.e500

Ninguno

Conflicto de intereses:

Ninguno que declarar.

Recibido: 22-8-2017

Aceptado: 2-1-2018
Cómo citar: Pennella CL, Rossi JG, Baialardo EM, et al. Leucemia linfoblástica aguda en niños con síndrome de down: análisis comparativo con pacientes sin síndrome de Down. Arch Argent Pediatr 2018;116(4):e500-e507.

\section{GLOSARIO}

ILE: Intervalo libre de eventos.

LLA:Leucemia linfoblástica aguda.

MRT: Muerte relacionada con el tratamiento.

RC: Remisión completa.

NSD: Sin síndrome de Down.

SD: Síndrome de Down.

SG: Sobrevida global.

SLE: Sobrevida libre de eventos.

\section{INTRODUCCIÓN}

Los pacientes con síndrome de Down (SD) tienen un riesgo aumentado 10-35 veces de desarrollar leucemias agudas. ${ }^{1,2} \mathrm{Si}$ bien estos pacientes conforman un grupo bien caracterizado de mejor pronóstico dentro de las leucemias mieloides agudas, los pacientes con SD y leucemia linfoblástica aguda (SDLLA) presentan una menor tasa de sobrevida respecto a los pacientes con LLA sin SD (NSD-LLA).

Los SD-LLA representan 3\% de las LLA..$^{3-5}$ Si bien no se han descrito diferencias significativas respecto a la demografía y clínica inicial, ${ }^{4,5}$ es inusual el diagnóstico en los niños menores de 1 año con SD. ${ }^{4,6}$ Los SDLLA presentan casi exclusivamente el inmunofenotipo precursor $B, y$ el inmunofenotipo $\mathrm{T}$ y $\mathrm{B}$ maduro es infrecuente..$^{5-7} \mathrm{El} 40 \%$ presenta cariotipo con bandeo-G normal vs. el $10-20 \%$ en los NSD-LLA. ${ }^{4,8}$ La menor sobrevida no se ha podido asociar 
a alteraciones citogenéticas recurrentes con implicancia pronóstica. ${ }^{3-5,7-10}$ Recientemente, la deleción del gen IKZF1 presente en el 30\% de los SD-LLA se asoció a inferior sobrevida. ${ }^{3,11-14}$

No se reportaron diferencias significativas en la respuesta temprana al tratamiento y la distribución por grupos de riesgo. ${ }^{5,15}$ Sin embargo, los pacientes con SD-LLA presentan mayores tasas de mortalidad ${ }^{15}$ con sobrevida global (SG) y sobrevida libre de eventos (SLE) 10-20\% menores que los pacientes NSD. El peor pronóstico se ha atribuido a una mayor morbimortalidad relacionada con el tratamiento (MRT) $)^{3,8}$ y mayor tasa de recaídas. ${ }^{8}$ No se han descrito segundas neoplasias en los pacientes con SD-LLA. ${ }^{4,15}$

La falta de conocimiento de las características de valor pronóstico en estos pacientes y la búsqueda de un balance entre menor MRT y tasa aumentada de recaídas generan serias dificultades sobre el mejor modo de adecuar la intensidad del tratamiento.

Con la finalidad de sumar herramientas que sirvan para la comprensión de este subgrupo de pacientes, el objetivo del presente estudio fue analizar las características clínicas, demográficasbiológicas y la respuesta al tratamiento de los pacientes con SD-LLA comparando con NSD-LLA.

\section{POBLACIÓN Y MÉTODOS}

Se realizó un estudio retrospectivo observacional. Se incluyeron, inicialmente, en el estudio, todos los pacientes con diagnóstico de LLA menores de 19 años admitidos desde enero de 1990 a noviembre de 2016 en el Hospital de Pediatría SAMIC "Prof. Dr. Juan P. Garrahan". Todos fueron enrolados en 4 protocolos sucesivos de tratamiento: LLA-90, LLA-96, Acute Lymphoblastic Leukemia Intercontinental (ALLIC) 2002 y ALLIC 2009. ${ }^{16,17}$ El tratamiento fue adaptado al grupo de riesgo de acuerdo con los criterios de cada estudio. En todos los casos, se realizó el diagnóstico por microscopía óptica, citometría de flujo, bandeo-G y reacción en cadena de la polimerasa con transcriptasa inversa (reverse transcription polymerase chain reaction; RT-PCR, por sus siglas en inglés) siguiendo los métodos previamente descritos para tales determinaciones. ${ }^{18-21}$

No se utilizaron criterios de exclusión para el análisis de las características clínicas y demográficas. Para el estudio de las características biológicas, se excluyeron los de edad menor de 1 año y, para el resto del trabajo, se consideraron evaluables los que no cumplieran los siguientes criterios: edad menor de un año, la utilización de tratamiento previo y la pérdida del seguimiento.

Las variables de interés buscadas fueron, para el análisis de las características clínicas y demográficas, el recuento inicial de leucocitos, compromiso extramedular al momento del diagnóstico, distribución por edad y sexo. Para el análisis de las características biológicas, inmunofenotipo, citogenética y alteraciones genéticas recurrentes. Se analizaron, además, los parámetros de respuesta temprana al tratamiento (respuesta a prednisona al día 8, remisión morfológica en la médula ósea al día 15), estratificación por grupos de riesgo (alto, estándar o bajo riesgo de falla de respuesta al tratamiento según los criterios utilizados en los protocolos de tratamiento), ${ }^{16,17} \mathrm{SLE}$, intervalo libre de eventos (ILE), SG y causas de mortalidad, que incluían MRT.

Se definieron como eventos las fallas en inducción, recaídas, segunda enfermedad maligna y muertes durante la inducción y en remisión completa (RC). Para el estudio de la SG, se utilizó como evento la muerte por cualquier causa.

Se definió como MRT toda muerte ocurrida en cualquier momento luego de haberse iniciado el tratamiento que no se relacionara con recaída o segunda enfermedad. Si la muerte se había producido en el curso de una infección documentada en forma clínica o microbiológica, se asumió de causa infecciosa.

Se utilizaron como métodos estadísticos los cálculos de chi cuadrado y el test Wilcoxonrank sum. La probabilidad de SLE, ILE y SG se analizaron utilizando el cálculo de Kaplan-Meier y sus comparaciones, con el test de log-rank.

El estudio fue aprobado por Comité de Ética del Hospital.

\section{RESULTADOS}

En el lapso reportado, se admitieron 1795 pacientes con diagnóstico de LLA menores de 19 años, de los cuales 54 (3\%) presentaban SD. La totalidad de esta población se utilizó para comparar las características clínicas y demográficas. Dado que ningún paciente con SD fue menor de 1 año vs. 109 pacientes NSDLLA, se excluyó a la población de infantes para el estudio de las características biológicas. Un paciente con SD-LLA se excluyó por haber recibido tratamiento previo. Para el resto de las variables analizadas, se evaluaron 53 pacientes con SD-LLA y 1491 pacientes NSD-LLA.

El análisis de las características clínicas y 
demográficas solo demostró diferencias estadísticamente significativas en la distribución por grupos etarios $(\mathrm{p}=0,0189)$ (Tabla 1, Figura 1), con una edad mediana mayor en los SD-LLA, debido a la ausencia de menores de un año. Tampoco se observaron diferencias significativas en el compromiso inicial de sitios extramedulares (evaluado con el compromiso inicial del sistema nervioso central mediante punción lumbar).

En el análisis comparativo de las características inmunofenotípicas y citogenéticas, se observó una diferencia significativa $(\mathrm{p}<0,0001)$ (Figura 2). En los
SD-LLA, no se detectaron anormalidades genéticas recurrentes asociadas a mal pronóstico, como la hipodiploidía, la translocación $\mathrm{t}(9 ; 22)$ y rearreglos del cromosoma 11q23. Las relacionadas con buen pronóstico, como la hiperdiploidía y la translocación $t(12 ; 21)$, se detectaron en menor proporción (11\% en SD-LLA vs. 30\% en NSD-LLA).

Con respecto a la trisomía adquirida en células leucémicas del cromosoma $21(+21)$, fue detectada en un $14,6 \%$ de los NSD-LLA precursor B y en un $49 \%$ de los NSD-LLA que presentaban hiperdiploidía.

TABla 1. Distribución de las características clínicas y demográficas de las leucemias linfoblásticas agudas en pacientes con sindrome de Down $(n=54)$ vs. leucemias linfoblásticas agudas en pacientes sin sindrome de Down $(n=1741)$

\begin{tabular}{|c|c|c|c|}
\hline & SD-LLA (n = 54) & NSD-LLA $(\mathrm{n}=1741)$ & Valor de $p$ \\
\hline $\begin{array}{l}\text { Edad: } \\
\text { Rango } \\
\text { Mediana } \\
<1 \text { año } \\
\text { De } 1 \text { a } 6 \text { años } \\
>6 \text { años }\end{array}$ & $\begin{array}{l}1 \text { a. y } 4 \mathrm{~m} \cdot-14 \text { a. y } 7 \mathrm{~m} . \\
5 \text { a. y } 9 \mathrm{~m} . \\
\mathrm{n}=0(0 \%) \\
\mathrm{n}=29(53,7 \%) \\
\mathrm{n}=25(46,3 \%)\end{array}$ & $\begin{array}{l}0 \text { a. y } 0 \text { m. }-16 \text { a. y } 11 \mathrm{~m} . \\
5 \text { a. y } 7 \mathrm{~m} . \\
\mathrm{n}=109(6,3 \%) \\
\mathrm{n}=819(47,0 \%) \\
\mathrm{n}=809(46,5 \%)\end{array}$ & $\mathrm{p}=0,0189$ \\
\hline $\begin{array}{l}\text { Sexo: } \\
\text { Femenino } \\
\text { Masculino }\end{array}$ & $\begin{array}{l}\mathrm{n}=23(42,6 \%) \\
\mathrm{n}=31(57,4 \%)\end{array}$ & $\begin{array}{l}\mathrm{n}=759(43,6 \%) \\
\mathrm{n}=980(56,4 \%)\end{array}$ & $\mathrm{p}=0,88$ \\
\hline \multicolumn{4}{|c|}{ Recuento de leucocitos } \\
\hline $\begin{array}{l}<20000 / \mathrm{mm}^{3} \\
>20000 / \mathrm{mm}^{3}\end{array}$ & $\begin{array}{l}\mathrm{n}=35(64,8 \%) \\
\mathrm{n}=19(35,2 \%)\end{array}$ & $\begin{array}{l}\mathrm{n}=1013(58,1 \%) \\
\mathrm{n}=725(41,9 \%)\end{array}$ & $\mathrm{p}=0,10$ \\
\hline
\end{tabular}

Leucemias linfoblásticas agudas en pacientes con síndrome de Down (SD-LLA); leucemias linfoblásticas agudas en pacientes sin síndrome de Down (NSD-LLA); años (a); meses (m).

FIGURA 1. Distribución por rango etario de las leucemias linfoblásticas agudas en pacientes con síndrome de Down ( $n=54)$ vs. leucemias linfoblásticas agudas en pacientes sin sindrome de Down $(n=1741)$

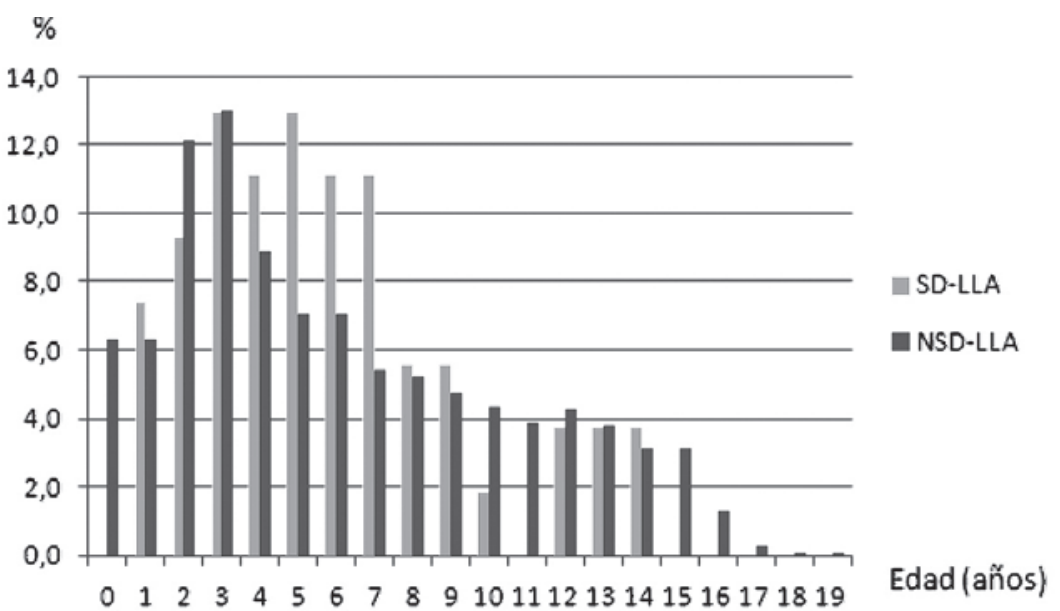

Leucemias linfoblásticas agudas en pacientes con síndrome de Down (SD-LLA); leucemias linfoblásticas agudas en pacientes sin síndrome de Down (NSD-LLA). 
En lo referido a la estratificación de los pacientes en grupos de riesgo pronóstico, se observó lo siguiente: riesgo alto de 7,4\% vs. $20,9 \%$; riesgo intermedio de $70,3 \%$ vs. $54,6 \%$; riesgo estándar de $22,3 \%$ vs. $24,5 \%$; SD-LLA vs. NSD-LLA, respectivamente $(p=0,036)$, lo que confirmó el menor porcentaje de pacientes con SD-LLA en el grupo de alto riesgo. En cuanto a los parámetros de respuesta temprana al tratamiento, los pacientes con SD-LLA presentaron una mejor tasa de respuesta temprana a la primera semana de tratamiento con prednisona como monodroga: 98\% en SD-LLA vs. $90 \%$ en NSD-LLA $(\mathrm{p}=0,09)$. En la evaluación de la médula ósea al día 15 intrainducción, se observó un $68 \%$ de pacientes con menos de $5 \%$ de blastos por microscopía óptica (criterio de RC por microscopía óptica) en SD-LLA vs. $64 \%$ en NSD-LLA ( $p=0,15)$.

En la evaluación de fin de inducción realizada al día 33, se observó una tasa significativamente menor de pacientes con SD-LLA que alcanzaron la $\mathrm{RC}(\mathrm{p}<0,0001)$ asociado al aumento de muertes en inducción (13\% en SD-LLA vs. $1,5 \%$ en NSDLLA) (Tabla 2). Luego de la RC, los pacientes fallecidos fueron $13 \%$ en SD-LLA vs. 3,9\% en NSD-LLA $(p=0,0074)$. El 100\% de las muertes en los SD-LLA fueron de causa infecciosa, mientras que, en los NSD-LLA, incluyeron otras causas, como síndrome de lisis tumoral agudo y hemorragias, entre las más frecuentes.

La tasa de recaídas fue similar en ambos grupos: $20,4 \%$ en SD-LLA vs. $19,7 \%$ en NSDLLA $(p=0,6894)$. No se observaron segundas neoplasias en los pacientes con SD-LLA.

La SLE y el ILE fueron significativamente menores en los pacientes con SD-LLA, $\mathrm{p}=0,006$ y $\mathrm{p}=0,0297$, respectivamente (Figura 3). La SG en SD-LLA fue de $49(+9) \%$. La media de seguimiento de los SD fue de 54 meses (rango: 15-189).

Dentro del grupo SD-LLA, se analizó el ILE clasificando a los pacientes de acuerdo con diferentes grupos de edades, y se observaron

FIGURA 2. Distribución por inmunofenotipo y anomalías citogenéticas recurrentes de las leucemias linfoblásticas agudas en pacientes con sindrome de Down $(n=54)$ vs. leucemias linfoblásticas agudas en pacientes sin sindrome de Down $(n=1632)$ mayores de un año

a. Distribución por inmunofenotipo

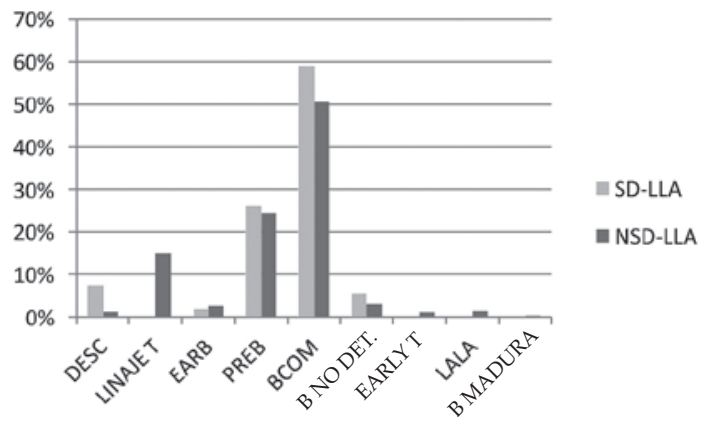

b. Distribución por anomalías citogenéticas recurrentes

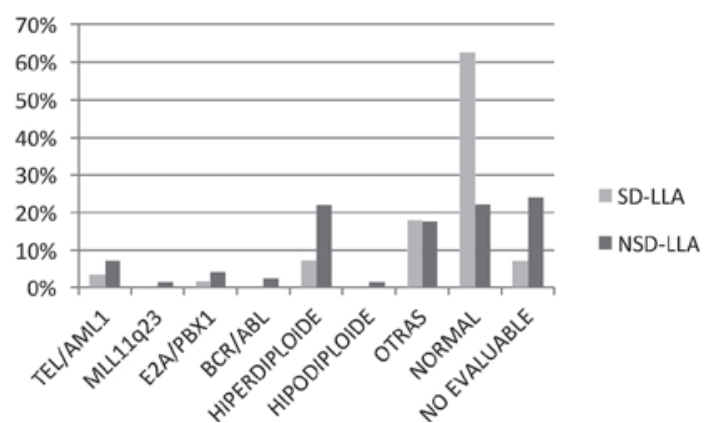

Leucemias linfoblásticas agudas en pacientes con síndrome de Down (SD-LLA); leucemias linfoblásticas agudas en pacientes sin síndrome de Down (NSD-LLA); pro-B (EARB): inmunofenotipo pro-B; pre-B (PREB): inmunofenotipo pre-B; B común (BCOM); $\mathrm{B}$ no determinado (B no det.); linaje ambiguo (LALA); desconocido (DESC.).

TABla 2. Respuesta a la inducción de las leucemias linfoblásticas agudas en pacientes con sindrome de Down $(n=53)$ vs. leucemias linfoblásticas agudas en pacientes sin síndrome de Down $(n=1491)$ evaluables mayores de 1 año

\begin{tabular}{lcccccc}
\hline Pacientes & RC & $\begin{array}{c}\text { SD-LLA (n= 53) } \\
\text { Muertes en inducción }\end{array}$ & M2/M3 & RC & $\begin{array}{c}\text { NSD-LLA (n= 1491) } \\
\text { Muertes en inducción }\end{array}$ & M2/M3 \\
\hline Número & 46 & 7 & 0 & 1446 & 22 & 23 \\
Porcentaje & 86,8 & 13,2 & 0 & 96,98 & 1,48 & 1,54 \\
\hline
\end{tabular}

Leucemias linfoblásticas agudas en pacientes con síndrome de Down (SD-LLA); leucemias linfoblásticas agudas en pacientes sin síndrome de Down (NSD-LLA); remisión completa (RC); médula ósea sin criterio de remisión completa, con > 5\% blastos por microscopía óptica (M2/M3). 
intervalos inferiores en los niños de 6 a 9 años $(\mathrm{p}=0,7885)$. También se observó una tendencia a una mejor SLE de los SD-LLA en los últimos 10 años de tratamiento, aunque la diferencia no resultó significativa $(\mathrm{p}=0,18)$ (Figura 4). Este resultado se asoció a la menor MRT de $40 \%$ vs. $15 \%$ y mayores tasas de RC de $45 \%$ vs. $64 \%$, protocolos LLA $90 / 96$ vs. ALLIC 2002/2009, respectivamente.

\section{DISCUSIÓN}

Tal como lo describe la literatura, en el presente estudio, se han hallado diferencias clínicas y biológicas entre los pacientes con SDLLA y NSD-LLA. Sin embargo, la mortalidad aumentada en los SD-LLA no se puede relacionar con diferencias en la prevalencia de algún factor utilizado en la actualidad para la estratificación de riesgo de sobrevida para esta patología, pero sí con la mayor mortalidad relacionada con el tratamiento.

Los pacientes con SD tienen riesgo aumentado de desarrollar leucemias agudas, con una incidencia para la LLA de 1/300 niños con SD vs. 1/3500 en NSD. ${ }^{1}$ Mientras que las leucemias mieloblásticas agudas en los niños con SD se caracterizan por la presencia de la mutación preleucémica adquirida del gen GATA-1, las bases genéticas que llevarían al desarrollo de los SD-LLA no son claras. Se postula que la trisomía constitucional del cromosoma 21 generaría una alteración de la hematopoyesis y alteraría tanto su

Figura 3. Curvas de probabilidad de sobrevida libre de eventos e intervalo libre de eventos de las leucemias linfoblásticas agudas en pacientes con síndrome de Down $(n=1491)$ y las leucemias linfoblásticas agudas en pacientes sin sindrome de Down $(n=53)$ evaluables mayores de un año

a. Curvas de probabilidad de sobrevida libre de eventos (SLE)

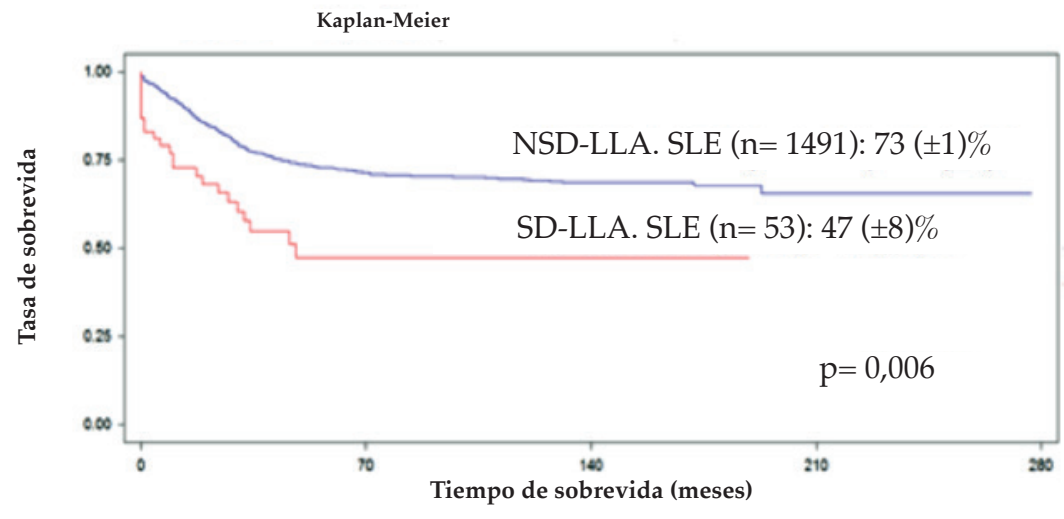

b. Curva de probabilidad de intervalo libre de eventos (ILE)

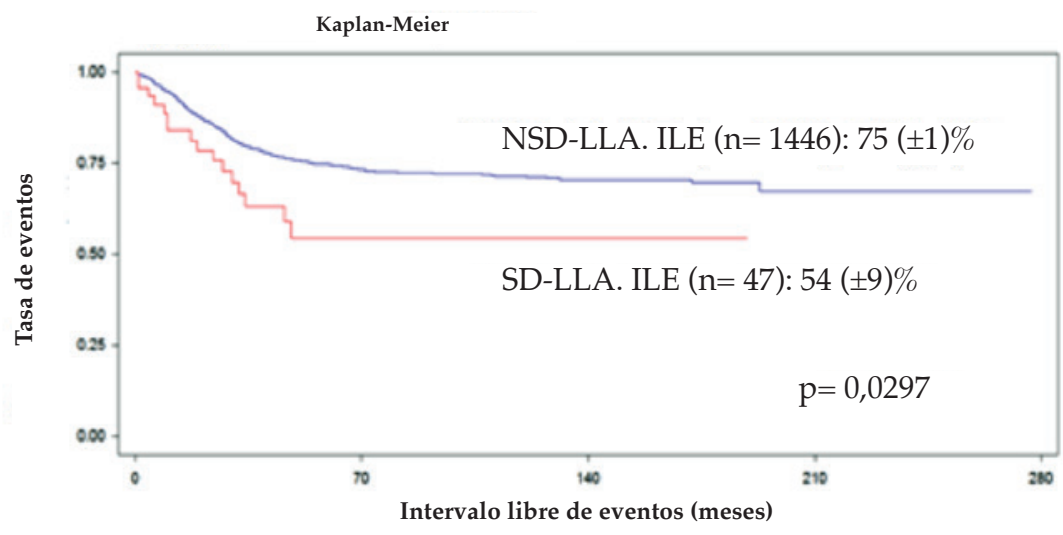

Leucemias linfoblásticas agudas en pacientes con síndrome de Down (SD-LLA); leucemias linfoblásticas agudas en pacientes sin síndrome de Down (NSD-LLA). 
microambiente como las células precursoras, que explicaría el aumento de incidencia de LLA en pacientes con SD. ${ }^{6,22}$ Interesantemente, la trisomía del cromosoma 21 es la anomalía numérica adquirida más común en las células leucémicas, que se presenta, tal como se observa en nuestro estudio, en el $20 \%$, ya sea sola o combinada. ${ }^{23}$

Las características clínicas y biológicas de los casos analizados en este estudio coinciden con lo descrito previamente en la literatura, así como la menor SLE e ILE en los pacientes con SD-LLA. Hay que tener presente que estos dos últimos hallazgos no pueden atribuirse a un mayor número de recaídas, sino a una incrementada tasa de MRT. La SG de los pacientes con SD-LLA fue similar a la SLE, explicable por la dificultad de rescatar a estos pacientes, dada la intensidad de tratamiento que se necesita para alcanzar una segunda remisión en las recaídas.

En los distintos protocolos para el tratamiento de la LLA pediátrica, se ha reportado una MRT de 2-4\%. En los pacientes con SD, se asoció la incrementada MRT tanto a la mayor toxicidad a la quimioterapia como a la mayor susceptibilidad a infecciones. Está descrito que la toxicidad gastrointestinal, la mucositis por metotrexato y las alteraciones hematológicas (mayor y más prolongada mielosupresión) son más frecuentes y graves en los pacientes con SD-LLA, comparativamente con los pacientes NSD-LLA. ${ }^{6,24-26}$ Sin embargo, se ha estudiado la citotoxicidad in vitro de los blastos de SD-LLA y

Figura 4. Curvas de probabilidad de intervalo libre de eventos según el rango etario y sobrevida libre de eventos según el protocolo de tratamiento de las leucemias linfoblásticas agudas en pacientes con síndrome de Down evaluables $(n=53)$

a. Curvas de probabilidad de intervalo libre de eventos (ILE) según el rango etario

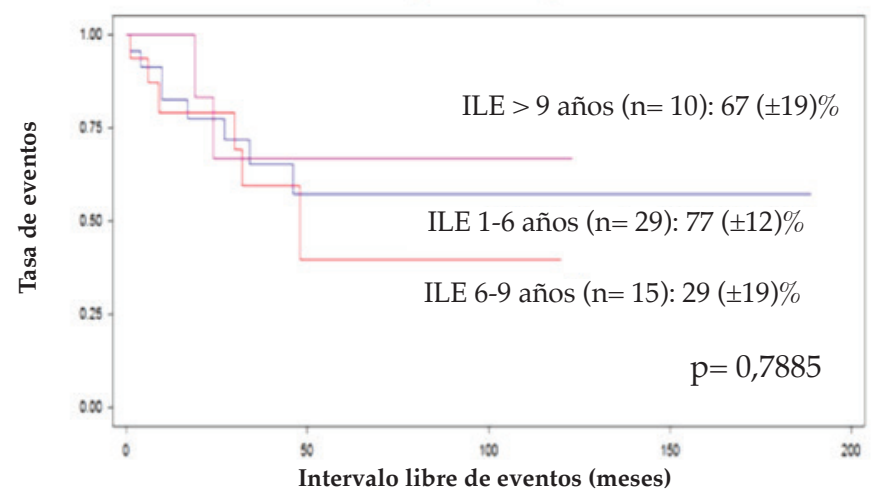

b. Curvas de probabilidad de sobrevida libre de eventos (SLE) según el protocolo de tratamiento

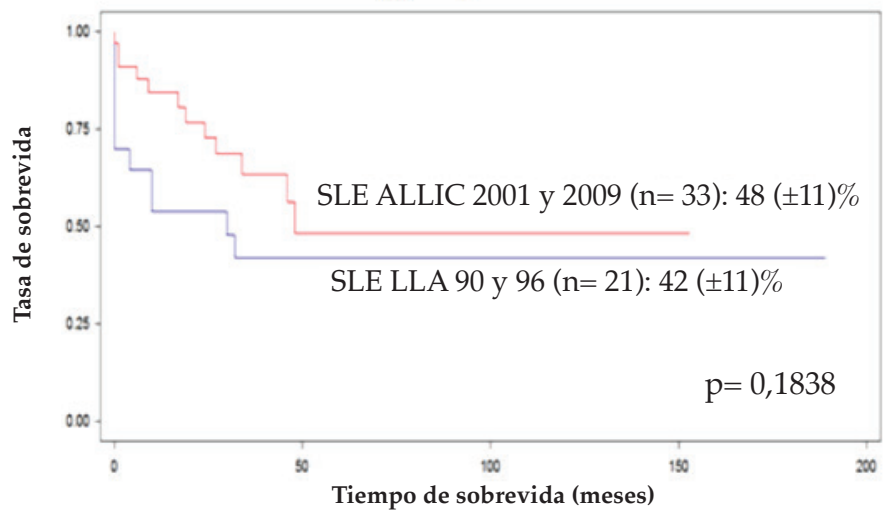

Leucemias linfoblásticas agudas en pacientes con síndrome de Down (SD-LLA); ALLIC: Acute Lymphoblastic Leukemia Intercontinental. 
no se ha encontrado mayor sensibilidad a distintos agentes quimioterápicos. ${ }^{27}$ Tampoco se ha observado una tasa aumentada de cardiotoxicidad asociada al uso de antraciclínicos. ${ }^{24}$ En nuestro estudio, no se ha observado MRT asociada exclusivamente a la toxicidad por quimioterapia, sino, en su totalidad, a infecciones, y estas muertes se presentan distribuidas en forma homogénea en todas las fases del tratamiento, tal como lo describe la literatura. ${ }^{3,8} \mathrm{La}$ inmunodeficiencia, junto con las alteraciones respiratorias y cardíacas que se asocian al SD, aumenta el riesgo de desarrollar complicaciones graves. Como se mencionó anteriormente, la trisomía constitucional del cromosoma 21 altera el desarrollo de los linfocitos B, lo que genera una moderada disgammaglobulinemia, además de provocar de moderada a grave disfunción de los linfocitos T. ${ }^{9}$ Las recomendaciones consensuadas para este grupo de pacientes sugieren un seguimiento clínico exhaustivo durante todo el tratamiento, inclusive el mantenimiento; mayores controles durante los períodos de neutropenia prolongada; tratamiento antibiótico agresivo durante la sospecha de infecciones, inclusive en ausencia de fiebre o neutropenia; inmunización contra influenza de la familia; tratamiento con gammaglobulinas endovenosas en los niños con hipogammaglobulinemia baja o moderada y profilaxis antibiótica. ${ }^{9}$

Distintas anomalías en la expresión génica fueron asociadas al peor pronóstico de los pacientes con SD. Aproximadamente, el $60 \%$ de los SD-LLA presentan sobreexpresión del receptor de citoquinas similar al factor 2 (cytokine receptorlike factor 2; CRLF2, por sus siglas en inglés) $)^{3,6,8,28}$ vs. $5 \%$ de los NSD-LLA, pero, a diferencia de lo que sucede en los NSD-LLA precursor $\mathrm{B},{ }^{29}$ en los SD-LLA, no tiene valor pronóstico, aun si se asocia a mutaciones de la quinasa JAK-2..$^{8,18,30,31}$ Las deleciones del gen IKZF1 presente en el 30\% de los SD-LLA ${ }^{11-14}$ han demostrado tener valor pronóstico adverso en este grupo de pacientes, con una SG aproximadamente $40 \%$ menor para los pacientes con deleción del gen. ${ }^{3,17}$ El grupo del estudio de Ponte di Legno ${ }^{8}$ asoció las diferencias en la SLE por grupo etario con la edad media de la población con deleción del gen IKZF1 y concluyó que la incidencia aumentada de esta mutación a mayor edad podría ser un factor genético que explicaría la menor SLE en los pacientes de 6-9 años. En nuestro reporte, se observó también una SLE menor en este grupo etario, pero no se pudo analizar la presencia de deleciones del gen
IKZF1 en nuestra población.

El riesgo aumentado de morbimortalidad observado en los pacientes con SD genera dificultades en el diseño de los esquemas de tratamiento por la necesidad de realizar adecuaciones de los distintos protocolos internacionales para tratar de lograr un equilibrio entre la mejoría de la SLE y SG y la disminución de la MRT. ${ }^{32}$ Es destacable que el $40-50 \%$ de los pacientes con SD requiere modificaciones del tratamiento (descenso de más del $20 \%$ de la dosis de quimioterapia o prolongación de más de $20 \%$ de las fases del tratamiento), lo que podría incidir negativamente en la sobrevida al aumentar las chances de recaídas. ${ }^{8,9,24,33}$ En los últimos protocolos de tratamiento utilizados en nuestro reporte, se ha observado una mejoría en la SLE relacionada con la menor MRT. Dado que las MRT no suceden en una fase particular del tratamiento, se ha concordado en que no se justificaría disminuir las dosis de quimioterapia. ${ }^{8}$ Sí existe consenso en la literatura sobre la necesidad de reducción de la dosis del metotrexato en el primer bloque de tratamiento con el aumento gradual según la tolerancia. ${ }^{9}$ Así mismo, la menor MRT, gracias al mejor soporte clínico en los últimos años, permitió el uso de tratamientos intensivos adecuados al riesgo, lo que aumentó el número alcanzado de pacientes en RC con SD-LLA.

Las perspectivas a futuro para este grupo de pacientes son la incorporación del análisis de nuevos factores de riesgo, como la mutación del gen IKZF1, y la necesidad de desarrollar guías institucionales de soporte clínico, siguiendo las últimas recomendaciones consensuadas en los grupos de referencia internacionales.

\section{CONCLUSIONES}

Este estudio confirma que los pacientes con SD-LLA presentan características clínicas y citogenéticas propias. Se corrobora que los pacientes con SD y edad comprendida entre 6 y 9 años tienen una tendencia a presentar sobrevida inferior.

Si bien los niños con SD-LLA tuvieron mejor respuesta inicial al tratamiento, la SLE e ILE fueron significativamente menores por la alta MRT, exclusivamente por sepsis.

\section{REFERENCIAS}

1. Lange B. The Management of Neoplastic Disorders of Haematopoeisis in Children with Down's Syndrome. Br J Haematol 2010;110(3):512-24.

2. Hasle H, Clemmensen IH, Mikkelsen M. Risks of leukaemia 
and solid tumours in individuals with Down's syndrome. Lancet 2000;355(9199):165-9.

3. Patrick K, Wade R, Goulden N, et al. Outcome of Down syndrome associated acute lymphoblastic leukaemia treated on a contemporary protocol. Br J Haematol 2014;165(4):552-5.

4. Lundin C, Forestier E, Klarskov Andersen M, et al. Clinical and genetic features of pediatric acute lymphoblastic leukemia in Down syndrome in the Nordic countries. J Hematol Oncol 2014;7:32.

5. Maloney KW, Carroll WL, Carroll AJ, etal. Down syndrome childhood acute lymphoblastic leukemia has a unique spectrum of sentinel cytogenetic lesions that influences treatment outcome: a report from the Children's Oncology Group. Blood 2010;116(7):1045-50.

6. Roberts I, Izraeli S. Haematopoietic development and leukaemiainDownsyndrome. BrJHaematol 2014;167(5):58799.

7. James R, Lightfoot T, Simpson J, et al. Acute leukemia in children with Down's syndrome: the importance of population based study. Haematologica 2008;93(8):1262-3.

8. Buitenkamp TD, Izraeli S, Zimmermann M, et al. Acute lymphoblasticleukemia in children with Down syndrome: a retrospective analysis from the Ponte di Legno study group. Blood 2014;123(1):70-7.

9. Izraeli S, Vora A, Zwaan CM, et al. How I treat ALL in Down's syndrome: pathobiology and management. Blood 2014;123(1):35-40.

10. Forestier E, Izraeli S, Beverloo B, et al. Cytogenetic features of acute lymphoblastic and myeloid leukemias in pediatric patients with Down syndrome: an iBFM-SG study. Blood 2008;111(3):1575-83.

11. Maloney KW, Taub JW, Ravindranath Y, et al. Down Syndrome Preleukemia and Leukemia. Pediatr Clin North Am 2015;62(1):121-37.

12. Hanada I, Terui K, Ikeda F, et al. Gene alterations involving the CRLF2-JAK pathway and recurrent gene deletions in Down syndrome-associated acute lymphoblastic leukemia in Japan. Genes Chromosomes Cancer 2014;53(11):902-10.

13. Attarbaschi A, Morak M, Cario G, et al. Treatment outcome of CRLF2-rearranged childhood acute lymphoblastic leukaemia: a comparative analysis of the AIEOP-BFM and UK NCRI-CCLG study groups. Br J Haematol 2012;158(6):772-7.

14. Buitenkamp TD, Pieters R, Gallimore NE, et al. Outcome in children with Down's syndrome and acute lymphoblastic leukemia: role of IKZF1 deletions and CRLF2 aberrations. Leukemia 2012;26(10):2204-11.

15. Arico M, Ziino O, Valsecchi MG, et al. Acute lymphoblastic leukemia and Down syndrome: presenting features and treatment outcome in the experience of the Italian Association of Pediatric Hematology and Oncology (AIEOP). Cancer 2008;113(3):515-21.

16. Felice M, Rossi J, Gallego M, et al. No Advantage of a Rotational Continuation Phase in Acute Lymphoblastic Leukemia in Childhood Treated With a BFM Back-Bone Therapy. Pediatr Blood Cancer 2011;57(1):47-55.

17. Stary J, Zimmermann $M$, Campbell $M$, et al. Intensive Chemotherapy for Childhood Acute Lymphoblastic Leukemia: Results of the Randomized Intercontinental Trial ALL IC-BFM 2002. J Clin Oncol 2014;32(3):174-84.

18. Swerdlow SH, Campo E, Harris NL, et al (eds). WHO Classification of Tumours of Haematopoietic and Lymphoid
Tissues. 4th ed. Lyon: IARC Press; 2008.

19. Bene MC, Castoldi G, Knapp W, et al. Proposal for the immunological classification of acute leukemias. European Group for the Immunological Characterization of Leukemias (EGIL). Leukemia 1995;9(10):1783-6.

20. Shaffer LG, Slovak ML, Campbell LJ, et al. ISCN. An International System for Human Cytogenetic Nomenclature. Basel, Switzerland: S. Karger; 2009.

21. VanDongenJJ,MacintyreEA, GabertJA, etal.Standardized RT-PCR analysis of fusion gene transcripts from chromosome aberrations in acute leukemia for detection of minimal residual disease. Report of the BIOMED-1 Concerted Action: investigation of minimal residual disease in acute leukemia. Leukemia 1999;13(12):1901-28.

22. De Vita S, Canzonetta C, Mulligan C, et al. Trisomic dose of several chromosome 21 genes perturbs haematopoietic stem and progenitor cell differentiation in Down's syndrome. Oncogene 2010;29(46):6102-14.

23. Malinge S, Izraeli S, Crispino JD. Insights into the manifestations, outcomes, and mechanisms of leukemogenesisinDownsyndrome. Blood2009;113(12):261928.

24. Shah N, Al-Ahmari A, Al-Yamani A, et al. Outcome and toxicity of chemotherapy for acute lymphoblasticleukemia in children with Down syndrome. Pediatr Blood Cancer 2009;52(1):14-9.

25. Rabin KR, Smith J, Kozinetz CA. Myelosuppression and infectious complications in children with Down syndrome and acute lymphoblastic leukemia. Pediatr Blood Cancer 2012;58(4):633-5.

26. Buitenkamp TD, MathôtRA, deHaas V, etal. Methotrexateinduced side effects are not due to differences in pharmacokinetics in children with Down syndrome and acute lymphoblastic leukemia. Haematologica 2010;95(7):1106-13.

27. Valle M, Plon SE, Rabin KR. Differential in vitro cytotoxicity does not explain increased host toxicities from chemotherapy in Down syndrome acute lymphoblastic leukemia. Leuk Res 2009;33(2):336-9.

28. EnsorHM, SchwabC, RussellLJ, etal.Demographic, clinical, and outcome features of children with acute lymphoblastic leukemia and CRLF2 deregulation: results from the MRC ALL97 clinical trial. Blood 2011;117(7):2129-36.

29. Harvey RC, Mullighan CG, Chen IM, et al. Rearrangement of CRLF2 is associated with mutation of JAK kinases, alteration of IKZF1, Hispanic/Latino ethnicity, and a poor outcome in pediatric B-progenitor acute lymphoblastic leukemia. Blood 2010;115(26):5312-21.

30. Russell LJ, Capasso M, Vater I, et al. Deregulated expression of cytokine receptor gene, CRLF2, is involved in lymphoid transformation in B-cell precursor acute lymphoblastic leukemia. Blood 2009;114(13):2688-98.

31. Kearney L, Gonzalez De Castro D, Yeung J, et al. Specific JAK2 mutation (JAK2R683) and multiple gene deletions in Down syndrome acute lymphoblastic leukemia. Blood 2009;113(3):646-8.

32. Maloney KW. Acute lymphoblastic leukaemia in children with Down syndrome: an updated review. Br J Haematol 2011;155(4):420-5.

33. Bohnstedt C, Levinsen M, Rosthøj S, et al. Physicians compliance during maintenance therapy in children with Down syndrome and acute lymphoblastic leukemia. Leukemia 2013;27(4):866-70. 